\title{
Radio Beacon Transponder System
}

National Cancer Institute

\section{Source}

National Cancer Institute. Radio Beacon Transponder System. NCI Thesaurus. Code C104907.

A tracking system that monitors the position of the target organ or tissue in real-time in order to spare surrounding tissues from radiation exposure during treatment. 